Research Article

\title{
Surgical presentations with abdominal pain in dengue fever
}

\author{
Shashirekha C. A.*, Sreeramulu P. N., Ravikiran H. R., Pawan Katti
}

Department of Surgery, Sri Devaraj Urs Medical College, Tamaka, Kolar, Karnataka, India

Received: 05 February 2016

Revised: 17 March 2016

Accepted: 31 March 2016

\section{*Correspondence:}

Dr. Shashirekha C. A.,

E-mail: drca_shashirekha@yahoo.co.in

Copyright: ( ) the author(s), publisher and licensee Medip Academy. This is an open-access article distributed under the terms of the Creative Commons Attribution Non-Commercial License, which permits unrestricted non-commercial use, distribution, and reproduction in any medium, provided the original work is properly cited.

\section{ABSTRACT}

Background: Dengue Fever (DF) is a tropical disease caused by single stranded RNA flavivirus that is transmitted by the bite of female Aedes aegypti mosquito. The objective of this study was to ascertain different etiologies for abdominal pain in dengue fever with their respective incidence and their management.

Methods: Patients admitted with dengue fever (confirmed by ELISA or NS1Ag) with pain abdomen in our hospital were included in the study. The cause of pain abdomen ascertained clinically and by following tests: Pancreatitis amylase, lipase, USG, CECT, Hepatitis-liver function test, Hollow viscous perforation Erect X-ray abdomen, Cholecystitis-USG, Appendicitis-TLC, USG.

Results: Out of total cases $(n=214)$ included into the study features of acalculous cholecystitis were seen in 122 $(58 \%)$ patients, pancreatitis in $24(11.5 \%)$, appendicitis in 4 (1.9\%), hepatitis: 27 (12.9\%) non-specific in 33 (15.7 $\%$ ) patients. All of the patients were managed conservatively and regularly followed up till they became asymptomatic. None of the patients required any surgical intervention. All the patients responded well to conservative line of management and were discharged.

Conclusions: Abdominal pain developing in dengue patients mimics many of surgical emergencies like cholecystitis, appendicitis, hepatitis, and pancreatitis. Most of these patients respond well to conservative line of management with I.V fluids, antibiotics, analgesics without the need for any surgical intervention. Hastily taken decisions with operative management for such patients have led to serious consequences in the past. Our study supports conservative line of management in such scenarios and advocates avoidance of surgical option. Our study also throws light on the various modes of presentations, with their respective percentages.

Keywords: DF, Pain abdomen, Acalculous cholecystitis, Pancreatitis, Hepatitis

\section{INTRODUCTION}

Dengue Fever (DF) is a tropical disease caused by single stranded RNA flavivirus that is transmitted by the bite of female Aedes aegypti mosquito. Dengue fever is usually non-specific and self-limiting, biphasic febrile illness but the presentation may range from being asymptomatic to dengue fever, dengue hemorrhagic fever and dengue shock syndrome. Typical dengue fever is characterized by high-grade fever, musculoskeletal pain, retro bulbar headaches, joint pain, nausea, vomiting and morbilliform rash. Headache and abdominal pain are common manifestations. ${ }^{1-4}$
Dengue fever is one of the commonest arbo-viral diseases around this part of the world with sudden insure in the number of cases observed during epidemics. In most of the cases it is asymptomatic, or presents with features common to any viral infection. Only some time though it presents with unusual presentations where it may be interpreted as a surgical emergency. Acute acalculus cholecystitis, hepatitis, pancreatitis, appendicitis are such atypical presentations, where patient presents with features typical of these conditions. On further investigations there is confirmation of the diagnosis. Now the dilemma: whether to go ahead with surgical management as is the norm or keeping in mind the complications associated with dengue fever like 
thrombocytopenia, hemorrhage, and shock to choose conservative line of management. ${ }^{5}$

\section{METHODS}

Patients admitted with dengue fever (confirmed by ELISA or NS1Ag) in our hospital and who developed pain abdomen was included in the study. The study period was from September 2013 to August 2014. The cause of pain abdomen ascertained clinically and based on the suspicion, patients were subjected to further appropriate investigations. Eg: when suspecting pancreatitis-serum amylase, lipase, USG abdomens were done, if required CECT abdomen.

Similarly for hepatitis-liver function test, USG abdomen, for hollow viscous perforation Erect X-ray abdomen, for cholecystitis-USG, for appendicitis-TLC, USG. Treatment given with antibiotics, intravenous fluids, analgesics, with regular monitoring for development of any further complications was done. The patients were followed till they recovered completely and discharged. Duration of admission was noted.

\section{RESULTS}

Out of total cases $(n=214)$ included into the study features of acalculous cholecystitis were seen in 122 (58 \%) patients, pancreatitis in $24(11.5 \%)$, appendicitis in $4(1.9 \%)$, hepatitis: 27 (12.9\%), nonspecific in $33(15.7 \%)$ patients (Table- 1$)$.

All of the patients were managed conservatively and regularly followed up till they became asymptomatic. Patients who had developed hepatitis took the longest time to recover, on average 8.6 days.

None of the patients required any surgical intervention. All the patients responded well to conservative line of management and were discharged following it without the need for any surgical management.

Table 1: Causes for pain abdomen in dengue fever.

\begin{tabular}{|llll|}
\hline Diagnosis & Total no. & percentage & Average duration of admission \\
\hline Acalculous cholecystitis & 122 & $58 \%$ & 7.8 days \\
\hline Pancreatitis & 24 & $11.5 \%$ & 8.2 days \\
\hline Appendicitis & 4 & $1.9 \%$ & 6 days \\
\hline Hepatitis & 27 & $12.9 \%$ & 8.6 days \\
\hline Non-specific & 33 & $15.7 \%$ & 5.6 days \\
\hline
\end{tabular}

\section{DISCUSSION}

Dengue fever is a common arbo-viral disease, presenting as febrile illness with constitutional symptoms. More serious form of presentation being-dengue hemorrhagic fever, dengue shock syndrome. Sometimes though the disease presents in an unusual mode of presentationfulminant hepatitis, encephalopathy, cardiomyopathy, acute pancreatitis, and acute acalculus cholecystitis.

The focus of our study was to find out the various modes of presentation of dengue fever with abdominal pain-the frequency, clinical features, prognosis, and clinical response of these conditions in dengue fever. Patients who were diagnosed with dengue fever, confirmed by IgM antibodies or NS1Ag antigen and who complained of pain abdomen were examined for the possible etiology for abdominal pain. Thorough physical examination with help of diagnostic investigations different conditions like acalculous cholecystitis, hepatitis, appendicitis, and pancreatitis were diagnosed.

In our study $58 \%$ of the patients were diagnosed with acalculous cholecystitis making it the most common cause which should be looked into when patient with dengue fever presents with abdominal pain in right hypochondriac region with tenderness in right hypochondriac region, murphy's sign positive confirmed by ultrasonographic findings of positive sonographic murphy's sign, pericholecystic fluid collection, thickened gall bladder wall. $12.9 \%$ of patients had hepatitis with increase in AST/ALT levels. Features of mild pancreatitis were seen in $11.5 \%$ of the patients with mild increase in serum amylase and lipase levels. A very small percentage of patients showed features of appendicitis $1.9 \%$. The second major groups $15.7 \%$ of the patients no exact cause could be defined probably because of limitation in the study as other investigations were not included into the study; these patients probably had other etiologies like gastric erosions or ulcerations which have been proved as cause in other studies. ${ }^{6,7}$

The pathophysiologic change in DF being - increased vascular permeability, causing plasma leakage and polyserositis. The endothelium is the target of the immunopathological mechanisms in dengue and DHF. This can explain involvement of such various body systems and the various myriad of presentations in dengue fever. ${ }^{8}$ 
All the patients in our study were managed conservatively with I.V fluids, analgesics, antipyretics and antibiotics. Patients were serially monitored for development of any complications or aggravation in the symptoms. Significantly all the patients responded very well to the conservative line of management with none of them requiring surgical intervention. This is of great significance as option of surgical intervention in such patients suffering from dengue may land up in complications because of factors such as thrombocytopenia, haemorrhage, shock which are frequently associated with dengue fever.

Thus we want to deduce that management of acute abdomen in dengue fever patients attributed to any of the etiology like cholecystitis, appendicitis, hepatitis, and pancreatitis is to be along conservative line.

\section{CONCLUSION}

Abdominal pain developing in dengue patients mimics many of surgical emergencies like cholecystitis, appendicitis, hepatitis, and pancreatitis. Most of these patients respond well to conservative line of management with I.V fluids, antipyretics, antibiotics, analgesics without the need for any surgical intervention. Hastily taken decisions with operative management for such patients have led to serious consequences in the past. Our study supports conservative line of management in such scenarios and advocates avoidance of surgical option. Our study also throws light on the various modes of presentations, with their respective percentages.
Funding: No funding sources Conflict of interest: None declared

Ethical approval: The study was approved by the institutional ethics committee

\section{REFERENCES}

1. World Health Organization. Dengue haemorrhagic fever: diagnosis, treatment, prevention and control. $2^{\text {nd }}$ ed. Geneva:WHO; 1997.

2. Khan E, Siddiqui J, Shakoor S, Mehraj V, Jamil B, HasanR. Dengue outbreak in Karachi, Pakistan, 2006: experience at a tertiary care centre. Trans R Soc Trop Med Hyg. 2007;101:1114-9.

3. Goh BK, Tan SG. Case of dengue virus infection presenting with acute acalculous cholecystitis. J Gastroenterol Hepatol. 2006;21:923-4.

4. Sharma N, Mahi S, Bhalla A, Singh V, Verma S, Ratho RK. Dengue fever related acalculous cholecystitis in a North Indian tertiary care hospital. JGastroenterol Hepatol. 2006;21:664-7.

5. Jaufeerally FR, Surrun SK, Chang PE. Acute acalculous cholecystitis in dengue hemorrhagic fever. Indian J Med Sci. 2007;61:613-4.

6. Wu KL, Changchien CS, Kou CM, Chuah SK, Lu $\mathrm{SN}$, EngHL et al. Dengue fever with acute acalculouscholecystitis. Am J Trop Med Hyg. 2003;68:657-60.

7. Wang JY, Tseng CC, Lee CS, Cheng KP. Clinical and upper gastro endoscopic features of patients with dengue virus infection. $\mathbf{J}$ Gastroenterol Hepatol. 1990;5:664-8.

8. Gulati S, Maheshwari A. Atypical manifestations of dengue. Tropical Medicine and International Health. 2007;12:1087-95.

Cite this article as: Shashirekha CA, Sreeramulu PN, Ravikiran HR, Katti P. Surgical presentations with abdominal pain in dengue fever. Int Surg J 2016;3:754-6. 